\title{
Les mesures atomiques de haute précision Un outil privilégié pour tester l'électrodynamique quantique
}

Pierre Cladé (pierre.clade@lkb.upmc.fr) et Lucile Julien (lucile.julien@lkb.upmc.fr) Laboratoire Kastler Brossel (Sorbonne Université, CNRS, ENS-Université PSL, Collège de France), 4 place Jussieu, 75005 Paris

À la fin des années 1940, l'étude des niveaux d'énergie de l'atome d'hydrogène et celle de l'anomalie du rapport gyromagnétique de l'électron ont été à l'origine de l'électrodynamique quantique.

Aujourd'hui encore, les mesures réalisées dans les systèmes atomiques simples, telles que celles que nous menons au laboratoire Kastler Brossel, permettent de tester les prédictions de cette théorie à un niveau de précision extrême qui, à ce jour, n'a pas réussi à la mettre en défaut.

\section{Des systèmes simples pour tester les prédictions de l'électrodynamique quantique}

L'électrodynamique quantique, née dans les années 1950, est la théorie qui décrit de façon quantique l'interaction des particules chargées avec le champ électromagnétique. Elle permet de calculer avec une précision extrême les niveaux d'énergie des systèmes atomiques simples et leurs propriétés lorsqu'ils interagissent avec un champ.

Constitué d'un proton et d'un électron, l'hydrogène est le plus simple des atomes. L'équation de Schrödinger permet de calculer sa fonction d'onde électronique et ses niveaux d'énergie. Pour un électron sans spin, cette énergie ne dépend que du nombre quantique principal $n$ et s'écrit $E=-R h c / n^{2}$, où $h$ et $c$ sont respectivement la constante de Planck et la vitesse de la lumière dans le vide, et $R$ la constante de Rydberg. Dans le cas limite où la masse du proton est supposée infinie comparée à celle de l'électron, cette constante a pour expression : $R_{\infty}=(1 / 2 h) m_{e} c \alpha^{2}(1)$. Dans cette formule, $m_{e}$ est la masse de l'électron et $\alpha$ la constante de structure fine, constante sans dimension qui décrit l'intensité du couplage électromagnétique. Cette constante vaut environ $1 / 137$, et s'écrit simplement $\alpha=e^{2} / 2 \varepsilon_{0} h c$, où $e$ est la charge élémentaire et $\varepsilon_{0}$ la permittivité du vide.

En 1928, Paul Dirac écrit une équation qui décrit l'électron dans un cadre à la fois quantique et relativiste. Appliquée à l'atome d'hydrogène, sa solution fait apparaître naturellement le spin de l'électron s comme un degré de liberté supplémentaire de sa fonction d'onde, qui n'est donc plus purement spatiale. L'énergie des niveaux dépend de $n$ et de $j$, nombre quantique associé à son moment cinétique total, somme de son moment cinétique orbital (décrit par le nombre quantique $l$ ) et de son moment cinétique de spin (décrit par s). La structure double de la raie $\mathrm{H} \alpha$ à $656 \mathrm{~nm}$, appelée aussi Balmer $\alpha$, qui relie les niveaux $n=2$ et $n=3$ de l'atome d'hydrogène, se trouve expliquée et l'écart calculé en bon accord avec l'expérience. En outre, l'équation de Dirac prédit une valeur de $-e / m_{e}$ pour le rapport gyromagnétique $\gamma$ de l'électron libre (voir encadré 1), valeur qui, à l'époque, semble conforme à l'observation et qui était inexpliquée jusqu'alors.

Dès les années 1930, les mesures spectroscopiques font progressivement soupçonner une structure plus complexe des niveaux $n=2$ de l'atome d'hydrogène. Alors que l'équation de Dirac prévoit la même énergie pour les niveaux $2 \mathrm{~S}_{1 / 2}(n=2, l=0$, $j=1 / 2)$ et $2 \mathrm{P}_{1 / 2}(n=2, l=1, j=1 / 2)$, ceci semble contredit par l'expérience. En 1947, Lamb et Retherford mesurent les fréquences de transitions radiofréquences entre niveaux $2 \mathrm{~S}$ et $2 \mathrm{P}$ de l'atome d'hydrogène en présence d'un champ magnétique [1]. Ils en déduisent qu'il y a, en champ nul, un écart d'environ $1 \mathrm{GHz}$ entre les niveaux $2 \mathrm{~S}_{1 / 2}$ et $2 \mathrm{P}_{1 / 2}$ : c'est le déplacement de Lamb (ou Lamb shift). Au même moment, l'étude 


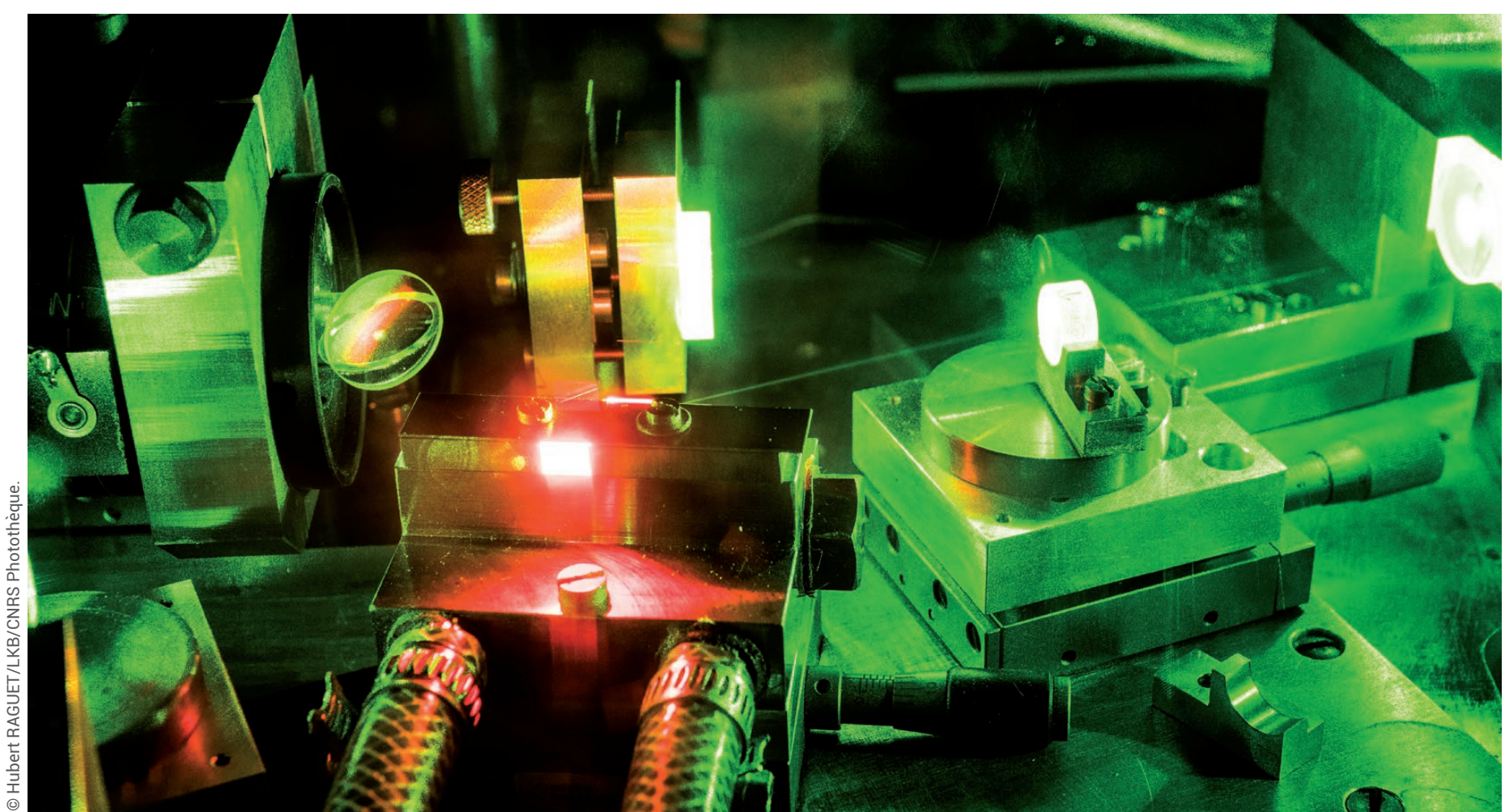

Expérience de spectroscopie à haute résolution de l'atome d'hydrogène (voir fig. 1, p. 7) : gros plan sur le cristal titane-saphir du laser d'excitation.

À toute particule possédant un moment cinétique $\vec{J}$, est associé un moment magnétique $\vec{M}=\gamma \vec{J}$. Le coefficient de proportionnalité $\gamma$ est appelé rapport gyromagnétique. La fréquence de précession angulaire du moment magnétique $\vec{M}$ dans un champ magnétique d'amplitude $B$, appelée fréquence de Larmor, est donnée par $\omega_{L}=-\gamma B$.

Pour un électron libre, le moment cinétique est purement de spin et l'équation de Dirac prédit que $\gamma=-e / m_{e}$ exactement. En réalité, dès les années 1930, on a soupçonné que cette valeur n'est pas parfaitement exacte et cela a été confirmé expérimentalement en 1948. On parle d'anomalie $a_{e}$ du moment magnétique de l'électron et on écrit : $\gamma=\left(1+a_{e}\right)\left(-e / m_{e}\right)$. Cette anomalie peut être calculée très précisément grâce à l'électrodynamique quantique. Expérimentalement, la mesure se fait en comparant la fréquence de Larmor et la fréquence cyclotron $\omega_{c}=\left(-e / m_{e}\right) B$ d'un électron dans un champ magnétique d'amplitude $B: a_{e}=\left|\omega_{L} / \omega_{c}\right|-1$.

La mesure la plus précise à ce jour est menée par G. Gabrielse et ses collaborateurs à Harvard. L'équipe utilise un piège magnétique de Penning cylindrique pour piéger un électron unique. L'ensemble est refroidi à $100 \mathrm{mK}$, de sorte que les états quantiques associés au mouvement de l'électron dans le piège sont résolus (fig. E1) et que l'électron est spontanément dans son état fondamental. On peut alors induire par un champ oscillant approprié soit la transition de l'électron vers son premier état excité à la fréquence cyclotron $\omega_{c}$, soit la transition de retournement de son spin à la fréquence de Larmor $\omega_{L}$. En observant les sauts quantiques associés à ces deux types de transition et en mesurant les deux fréquences $\omega_{c}$ et $\omega_{L}$, les chercheurs de Harvard ont obtenu ainsi une précision de $3 \times 10^{-10}$ sur $a_{e}[7]$.

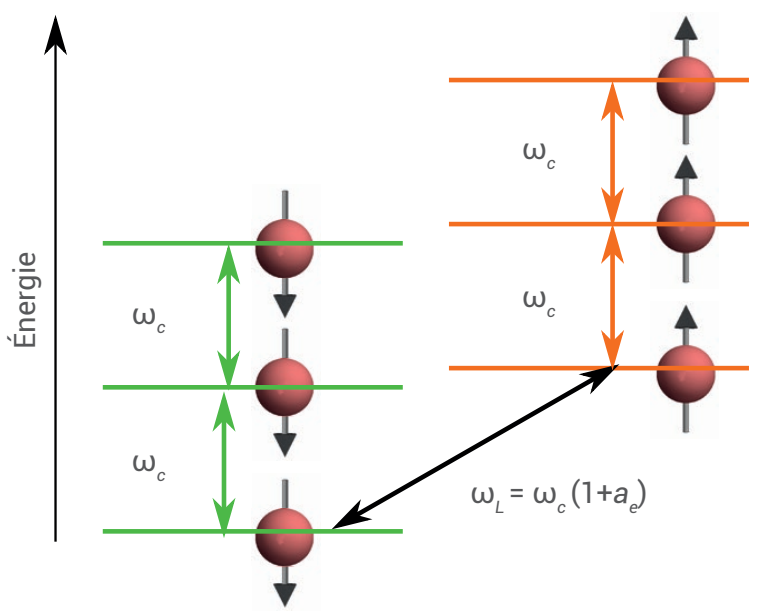

E1. Niveaux d'énergie d'un électron unique dans un champ magnétique. 
$>>$

de différents états atomiques en présence d'un champ magnétique amène à la conclusion que le rapport gyromagnétique de l'électron est légèrement supérieur à la valeur attendue. On parle d'anomalie $a_{e} \mathrm{du}$ moment magnétique de l'électron, définie $\operatorname{par} \gamma=\left(1+a_{e}\right)\left(-e / m_{e}\right)$.

La théorie de l'électron de Dirac est incomplète car, comme l'atome, le champ électromagnétique doit être traité quantiquement. Même isolé, un atome interagit avec les fluctuations du champ électromagnétique, ce qui a pour effet de déplacer ses niveaux d'énergie. L'électrodynamique quantique permet de calculer ces corrections radiatives par un calcul perturbatif en puissances de $\alpha$, en particulier le déplacement de Lamb et l'anomalie du rapport gyromagnétique de l'électron. Dès les années 1950, les résultats obtenus sont en accord avec les expériences. Soixante ans après, la confrontation théorie-expériences continue et s'accompagne de progrès spectaculaires tant au niveau théorique qu'expérimental.

Notre équipe de métrologie au Laboratoire Kastler Brossel est impliquée à la fois dans la mesure de la constante de structure fine et dans la spectroscopie de l'atome d'hydrogène. Dans les deux cas, nos résultats permettent de tester les prédictions de l'électrodynamique quantique à un très haut niveau de précision, soit sur le moment magnétique de l'électron libre, soit sur les niveaux d'énergie de l'atome d'hydrogène.

\section{La spectroscopie de l'atome d'hydrogène}

Les corrections d'électrodynamique quantique affectent tous les niveaux d'énergie de l'atome d'hydrogène, mais leur effet est le plus important dans les niveaux $\mathrm{S}$, de moment cinétique orbital nul $(l=0)$. En outre, le déplacement de Lamb associé varie à peu près comme $1 / n^{3}$, ce qui fait que le niveau fondamental $1 \mathrm{~S}$ de l'hydrogène a une énergie accrue d'environ $8 \mathrm{GHz}$ par rapport aux prévisions de l'équation de Dirac.

Si la première mesure du Lamb shift a été réalisée sur l'écart $2 \mathrm{~S}_{1 / 2}-2 \mathrm{P}_{1 / 2}$, c'est que cette transition se situe dans le domaine des radiofréquences, facile à atteindre avec les techniques d'après-guerre. Actuellement, ce sont les méthodes optiques qui sont les plus précises grâce aux lasers et aux peignes de fréquences réalisés avec des lasers femtosecondes [2]. L'étude de transitions à deux photons, dans lesquelles les atomes sont excités par deux faisceaux lasers contra-propageants (de vecteurs d'onde opposés), permet d'éliminer l'effet Doppler du premier ordre en faisant absorber aux atomes un photon de chaque onde.

Pour déterminer à la fois la constante de Rydberg $R_{\infty}$ et le déplacement de Lamb $L_{1 S}$ de l'état $1 \mathrm{~S}$, on compare simplement deux fréquences de transition optiques, dont l'une au moins concerne le niveau fondamental 1S. Deux données expérimentales sont suffisantes pour déterminer les deux constantes, car les autres déplacements de Lamb qui interviennent aussi dans les fréquences de transition sont beaucoup plus petits et suffisamment connus.

La transition la plus précisément mesurée est la transition 1S-2S à $243 \mathrm{~nm}$, dont la largeur naturelle est de l'ordre du hertz, grâce à la très longue durée de vie du niveau 2S. L'incertitude relative atteinte sur la mesure de sa fréquence, réalisée dans l'équipe de T.W. Hänsch à Garching (Allemagne) est de $4,2 \times 10^{-15}$ [3]. Les fréquences de transition $2 \mathrm{~S}-n \mathrm{~S}$ et $2 \mathrm{~S}-n \mathrm{D}$ ( $n=8$ et $n=12)$, qui ont été mesurées par notre équipe, jouent un rôle prépondérant pour la détermination de $R_{\infty}$ et $L_{1 \mathrm{~s}}$ par comparaison avec la fréquence de la transition 1S-2S. La constante de Rydberg en est actuellement déduite avec une incertitude meilleure que $6 \times 10^{-12}$ en valeur relative. Quant à la précision sur le déplacement $L_{1 \mathrm{~S}}$, elle serait suffisante pour tester les calculs d'électrodynamique quantique jusqu'à l'ordre 4 en $\alpha$, si le rayon du proton $r_{\mathrm{p}}$ était parfaitement connu (encadré 2).

Le fait que la distribution de charge du proton n'est pas ponctuelle rehausse en effet les niveaux d'énergie de l'atome d'hydrogène et particulièrement les niveaux S pour lesquels la probabilité de présence de l'électron près du proton est grande. Cet effet varie en $1 / n^{3}$ comme les corrections d'électrodynamique quantique, et contribue donc au déplacement de

Le rayon du proton et son " mystère » encadré 2

Le proton n'est pas une particule élémentaire, contrairement à l'électron. Il est constitué de trois quarks. En conséquence, sa répartition de charge possède un certain volume qu'on caractérise par la quantité $r_{\mathrm{p}}=\left(\int_{0}^{\infty} r^{2} \rho(r) d^{3} r / \int_{0}^{\infty} \rho(r) d^{3} r\right)^{1 / 2}$, appelée rayon de charge du proton. Ce rayon est compris entre 0,8 et $0,9 \mathrm{fm}\left(1 \mathrm{fm}=10^{-15} \mathrm{~m}\right)$.

Les déterminations de $r_{\mathrm{p}}$ sont obtenues soit par diffusion d'électrons sur des protons, soit par spectroscopie. Pour améliorer la précision sur $r_{\mathrm{p}}$ et pouvoir ainsi tester l'électrodynamique quantique sur les énergies des niveaux de l'atome d'hydrogène (e-p), la collaboration internationale CREMA (Charge Radius Experiment with Muonic Atoms), dont nous faisons partie, s'est mise en place autour du Paul Scherrer Institute (PSI) en Suisse. Son premier but était de mesurer le déplacement de Lamb dans I'hydrogène muonique $(\mu-p)$, un atome d'hydrogène dans lequel l'électron est remplacé par un muon de charge électrique négative. D'après le modèle standard de la physique des particules, ce muon a des propriétés identiques à celles de l'électron, exceptée sa masse qui se trouve être 207 fois plus grande.

La mesure de deux des transitions 2S-2P de l'hydrogène muonique à $6 \mu \mathrm{m}$ a été réalisée en 2009. Nous avons pu en déduire le rayon de charge du proton en utilisant la valeur de la constante de Rydberg et les calculs de l'électrodynamique quantique. La valeur obtenue est $r_{p}=0,84087(39) \mathrm{fm}$; elle est plus précise par un ordre de grandeur que celle déduite des mesures précédentes, mais plus petite de $7 \sigma$ (7 écarts-types) [5]. Ce désaccord est à l'origine de ce qu'on appelle aujourd'hui le « mystère du proton ». Ces dernières années, une intense activité théorique a cherché à résoudre ce « mystère ». Parmi toutes les hypothèses envisagées, aucune ne s'est révélée concluante dans le cadre du modèle standard. 
Lamb observé. C'est pourquoi la connaissance de $L_{1 \mathrm{~s}}$ peut servir soit à tester le calcul des corrections radiatives en admettant une valeur donnée pour $r_{\mathrm{p}}$, soit à déterminer ce rayon en supposant ce calcul exact.

Le « mystère du proton " (encadré 2) incite à améliorer encore les mesures des fréquences de transition de l'atome d'hydrogène. Dans notre équipe, nous étudions actuellement la transition à deux photons 1S-3S, qui est déjà la deuxième transition la mieux connue dans l'atome d'hydrogène grâce à nos travaux [4]. La transition est excitée dans un jet atomique, à l'intérieur d'une cavité de surtension dont l'axe est colinéaire au jet (fig. 1).

Les deux points clés de cette expérience sont la source laser à $205 \mathrm{~nm}$, utilisée pour exciter la transition, et la mesure absolue de la fréquence correspondante. Notre source est la seule au monde qui soit continue et ajustable dans cette gamme de longueur d'onde. Elle est obtenue par somme de fréquences dans un cristal non linéaire de $\mathrm{BBO}\left(\beta-\mathrm{BaB}_{2} \mathrm{O}_{4}\right)$, entre la radiation issue d'un laser titane-saphir à $894 \mathrm{~nm}$, mis au point par notre équipe, et celle d'un laser commercial à $266 \mathrm{~nm}$ (laser solide à $1064 \mathrm{~nm}$, doublé deux fois en fréquence).

La figure 1 représente les différents lasers de notre dispositif, utilisés pour l'excitation et pour la mesure. Différents éléments ne sont pas représentés sur la figure, en particulier un laser de référence et des cavités Fabry-Perot qui, grâce à des asservissements, permettent de stabiliser la fréquence du laser titane-saphir et de balayer celle-ci de façon reproductible. À la sortie du dispositif de somme de fréquences, nous obtenons plus de $10 \mathrm{~mW}$ à $205 \mathrm{~nm}$. Cette puissance est suffisante pour enregistrer le signal de transition $1 \mathrm{~S}-3 \mathrm{~S}$ avec un rapport signal-àbruit de 170 après 3,5 h d'intégration. La fréquence de la transition est déterminée en mesurant simultanément les fréquences des deux radiations à 894 et 266 nm, par comparaison avec un peigne de fréquences réalisé avec un laser femtoseconde [1]. Le peigne est étalonné à l'aide d'un signal de référence à $100 \mathrm{MHz}$ qui nous est envoyé par le LNE-SYRTE (Observatoire de Paris) à travers une fibre de $3 \mathrm{~km}$ de longueur. En pratique, nous mesurons en permanence pendant l'enregistrement du signal deux fréquences de battement à 1064 et 894 nm avec deux dents du peigne, dont les fréquences sont bien connues.

Avec un tel dispositif, nous avons mesuré récemment la fréquence de la transition 1S-3S avec une incertitude de $2,6 \mathrm{kHz}$, soit $9 \times 10^{-13}$ en valeur relative [6], alors que pour cette transition l'écart associé aux deux valeurs contradictoires du rayon du proton est de $7 \mathrm{kHz}$. Les calculs d'électrodynamique quantique permettent de déduire de notre mesure une valeur de ce rayon de $r_{p}=0,877(13) \mathrm{fm}$, en désaccord avec celle déduite de l'hydrogène muonique $\left(r_{p}=0,84087(39) \mathrm{fm}\right.$, voir l'encadré 2$)$. Le " mystère du proton " n'est pas encore éclairci...

\section{Mesure de la constante de structure fine}

Comme pour celui du Lamb shift, le calcul de l'anomalie $a_{\mathrm{e}}$ du moment magnétique de l'électron (voir l'encadré 1) grâce à l'électro-

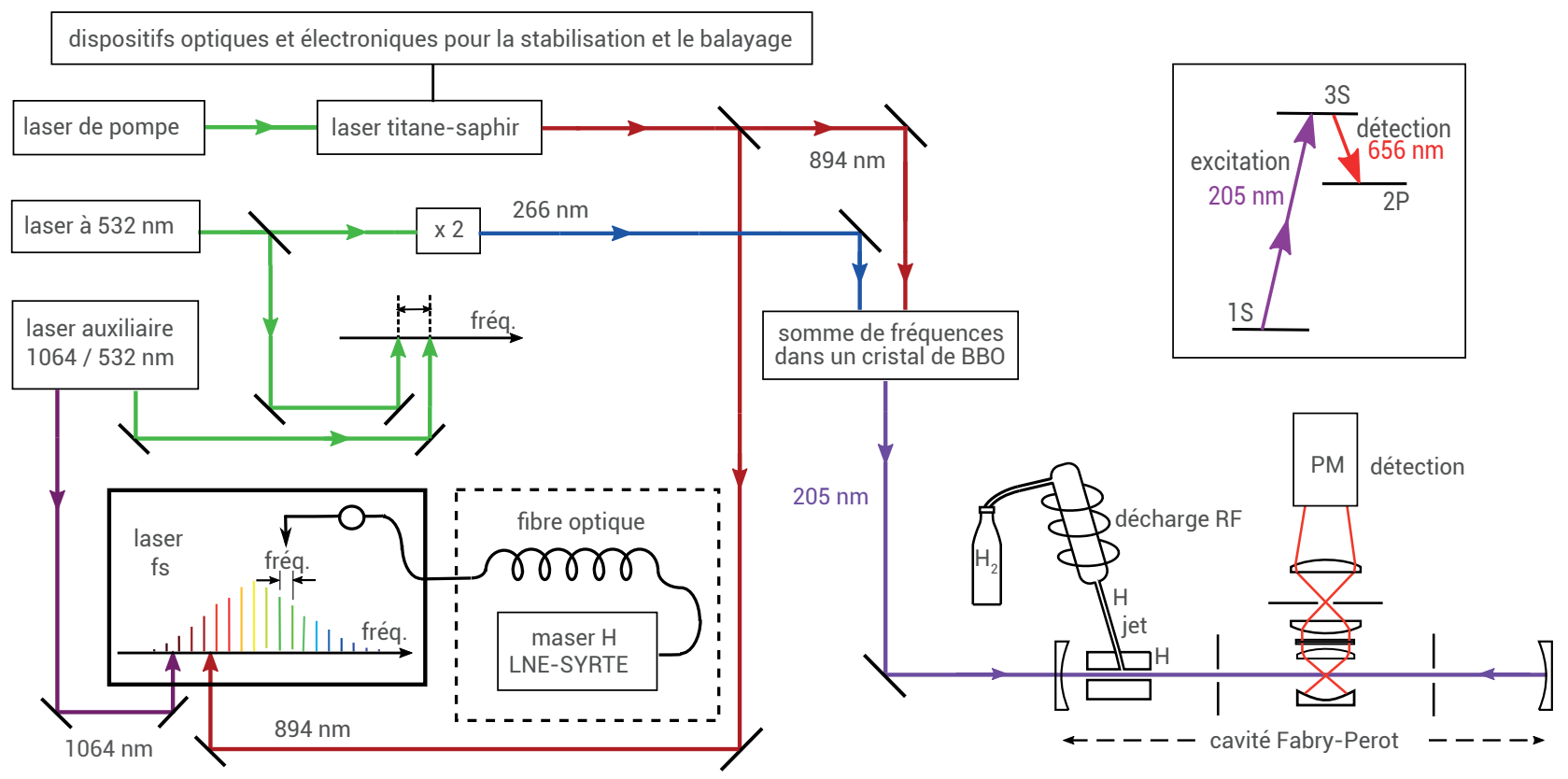

1. Dispositif expérimental pour l'excitation et la mesure en fréquence de la transition $1 \mathrm{~S}-3 \mathrm{~S}$ à $205 \mathrm{~nm}$ de l'atome d'hydrogène : le faisceau excitateur est obtenu par somme de fréquences à partir d'un laser titane-saphir accordable à $894 \mathrm{~nm}$ et d'un laser à $266 \mathrm{~nm}$. Ce dernier est calibré par rapport à un laser à $1064 \mathrm{~nm}$. Les deux fréquences des radiations à $894 \mathrm{~nm}$ et $1064 \mathrm{~nm}$ sont mesurées simultanément par comparaison avec un peigne de fréquences femtoseconde référencé sur les horloges du LNE-SYRTE. Les molécules de dihydrogène sont dissociées dans une décharge radiofréquence et l'excitation laser à deux photons est réalisée sur un jet effusif d'hydrogène, dans une cavité Fabry-Perot ; elle est détectée sur le signal de fluorescence à $656 \mathrm{~nm}$ (transition Balmer $\alpha$, entre les niveaux $n=2$ et $n=3$ ). 


\section{Principe de l'expérience de mesure de la vitesse de recul}

Notre interféromètre atomique utilise la technique de Ramsey, développée à l'origine dans le domaine des radiofréquences, et qui permet d'obtenir une très grande sensibilité. Une première impulsion Raman entre deux niveaux hyperfins différents de l'atome de rubidium (flèches rouges et bleues sur la figure E2a) sépare le nuage d'atomes froids en deux paquets d'ondes atomiques de vitesses différentes. Elle est suivie d'une deuxième impulsion après laquelle les deux paquets ont même vitesse mais sont distants l'un de l'autre. À la sortie de l'interféromètre, une deuxième paire d'impulsions Raman est appliquée qui superpose à nouveau spatialement les deux paquets d'onde en refermant donc l'interféromètre à atomes.

Entre les deux paires d'impulsions, c'est-à-dire pendant son trajet dans l'interféromètre, chaque paquet est accéléré. Cette accélération est effectuée par une série de transitions Raman représentées par des flèches orange, transférant chacune deux fois la vitesse de recul $v_{r}$ aux atomes.

Les deux sorties de l'interféromètre correspondent aux deux états internes de l'atome $(F=1$ et $F=2)$. On mesure la proportion relative d'atomes dans un état en fonction de la différence de fréquence $\delta_{\text {sel }}-\delta_{\text {mes }}$ entre les impulsions utilisées pour la sélection et celles utilisées pour la mesure. Lorsque cette différence de fréquence compense l'effet Doppler induit par l'accélération, la différence de phase entre les deux chemins est nulle et on observe la frange centrale (fig. E2c).

Interféromètre atomique

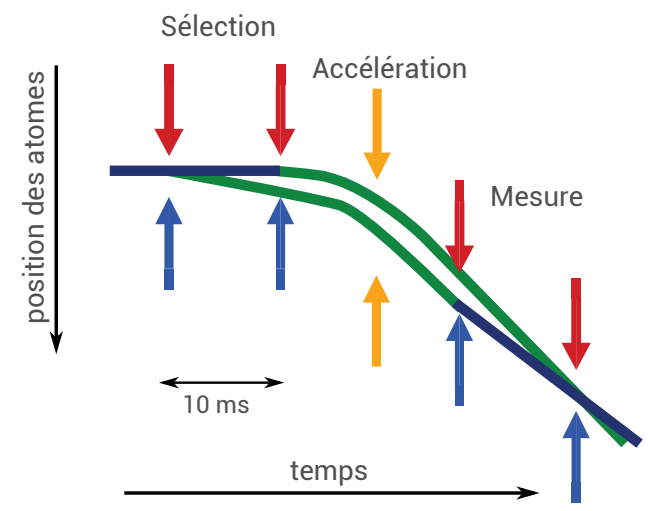

a

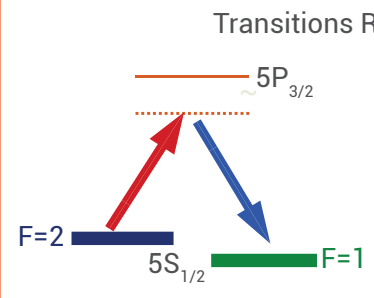

Sélection et mesure

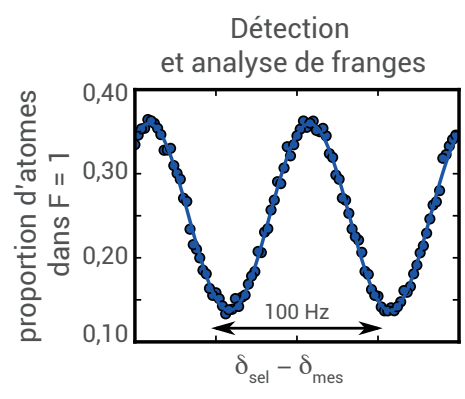

c
E2. Mesure par interférométrie atomique de la vitesse de recul d'atomes de rubidium soumis à des impulsions Raman.

(a) Déplacement des deux paquets d'ondes en fonction du temps

(b) Transitions Raman stimulées.

(c) Observation de la frange centrale.

\section{$\gg>$}

dynamique quantique, fait intervenir un développement en puissances de $\alpha$. La somme des termes en $\alpha^{5}$ a été calculée pour la première fois en 2012 : elle fait intervenir pas moins de 12672 intégrales et contribue à hauteur d'environ $10^{-9}$ à l'anomalie $a_{\mathrm{e}}$. À ce niveau, elle est comparable à d'autres corrections qui ne sont pas d'origine électronique : celle due aux forces électrofaibles, ainsi que les corrections radiatives liées aux autres leptons (principalement les muons) et aux hadrons.

Afin de pouvoir tester la valeur prédite de $a_{e}$ avec une précision donnée, il est nécessaire de connaître la valeur de la constante de structure fine $\alpha$ avec cette même précision. Une bonne méthode pour déterminer directement $\alpha$ consiste à s'appuyer sur la relation (1) de la page 4 Comme $c$ a une valeur fixée et que $R_{\infty}$ est connue expérimentalement avec une incertitude inférieure à $10^{-11}$, la mesure de $\alpha$ peut se ramener à celle du rapport entre la constante de Planck $h$ et la masse de l'électron $m_{e}$.

La méthode utilisée par notre équipe pour mesurer le rapport $h / m_{e}$ consiste à mesurer la vitesse de recul d'un atome de rubidium lorsqu'il absorbe un photon se propageant dans une direction donnée. En raison de la conservation de l'impulsion totale dans le processus d'absorption, cette vitesse de recul vaut : $v_{\mathrm{r}}=h \boldsymbol{v} / m c$ où $v$ est la fréquence du photon et $m$ la masse de l'atome de rubidium. Pour un photon dans le proche infrarouge, elle vaut environ $6 \mathrm{~mm} / \mathrm{s}$. On déduit de cette mesure le rapport $h / m$. Sachant que les méthodes de comparaison de masses dans des pièges cyclotrons permettent de connaître les rapports de masses atomiques et le rapport $m_{\mathrm{e}} / m$ avec une précision meilleure que $10^{-10}$, on comprend que cette méthode donne accès à une détermination de $\alpha$ avec un niveau de précision de l'ordre de $10^{-10}$

Le principe de la mesure de la vitesse de recul, décrit dans l'encadré 3, est le suivant : on transfere à un nuage d'atomes de rubidium, refroidi par laser à quelques microkelvins, un grand nombre de fois la vitesse de recul $v_{\mathrm{r}}$ et on mesure le changement de vitesse subi par les atomes. Pour réaliser ces deux tâches, on utilise un outil commun : les transitions Raman dans lesquelles l'atome absorbe et émet simultanément un photon, à partir d'un des deux niveaux hyperfins $(F=1$ et $F=2)$ de son 
état fondamental. Si les faisceaux lasers utilisés sont contrapropageants, ce qui est le cas dans notre expérience, les deux vitesses de recul transmises aux atomes par la transition Raman s'ajoutent (absorption d'un photon d'une direction et émission stimulée d'un photon dans l'autre direction). Contrairement aux transitions à deux photons vues dans la partie précédente où les deux photons étaient absorbés et où les effets Doppler se compensaient, les effets Doppler s'ajoutent ici et la transition est donc sélective en vitesse.

Pour transférer un grand nombre de reculs, nous utilisons des transitions Raman qui laissent les atomes dans le même état interne. Pour induire successivement plusieurs transitions, il est nécessaire de corriger la fréquence des lasers de façon à maintenir les atomes à résonance en compensant l'effet Doppler dû au recul. Cela se fait en balayant linéairement la différence de fréquence entre les deux lasers, de sorte que les deux faisceaux forment un réseau optique uniformément accéléré qui entraîne les atomes. Avec notre enceinte à vide et nos lasers, nous pouvons réaliser sur un nuage atomique un grand nombre de transitions (absorption + émission), et transférer aux atomes jusqu'à 1000 fois la vitesse de recul $v_{\mathrm{r}}$. Pour sélectionner et mesurer la vitesse des atomes, on utilise des transitions Raman contrapropageantes qui changent l'état interne des atomes (encadré 3). En les faisant intervenir lors d'une séquence temporelle bien choisie, on réalise un dispositif d'interférométrie atomique avec deux paquets d'onde associés aux atomes froids [8].

$\mathrm{Ce}$ dispositif permet de mesurer un changement de vitesse atomique avec une résolution inversement proportionnelle à la distance entre les deux paquets dans l'interféromètre. Nous sommes capables dans notre expérience d'obtenir une séparation de $120 \mu \mathrm{m}$ en $10 \mathrm{~ms}$, ce qui permet d'observer des franges de largeur $100 \mathrm{~Hz}$ (fig. E2c), correspondant à une résolution en vitesse de $40 \mu \mathrm{m} / \mathrm{s}$. Compte tenu du rapport signal-à-bruit et en moyennant un grand nombre de mesures, on peut atteindre une sensibilité de l'ordre de $10^{-9}$ sur le rapport $h / m_{\mathrm{e}}$.

Avec cette méthode, nous avons, en 2010, déterminé $\alpha$ avec une incertitude inférieure à $7 \times 10^{-10}$ [9]. La valeur de l'anomalie $a_{\mathrm{e}}$ peut en être déduite et le résultat obtenu comparé à celui du groupe d'Harvard (fig. 2). L'accord entre les deux résultats est

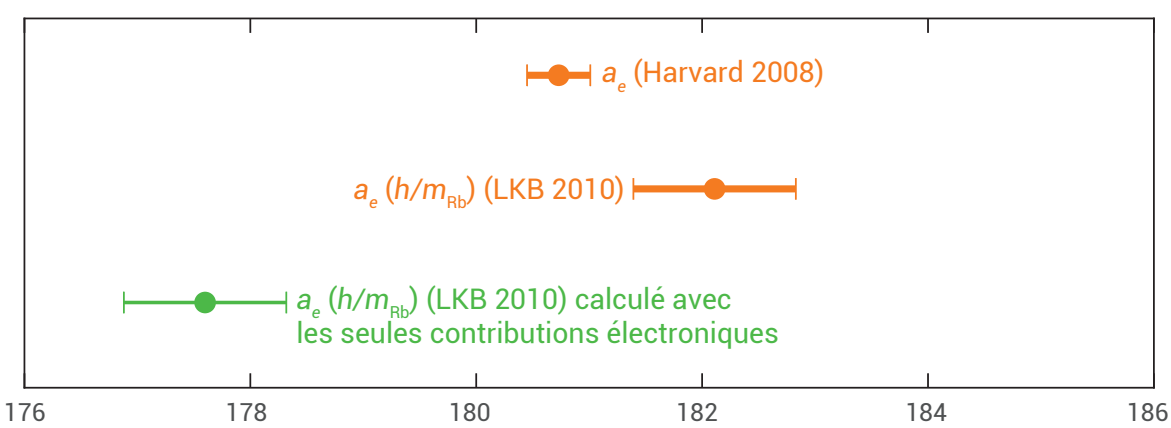

2. Comparaison des valeurs de $a_{e}$ obtenues expérimentalement par le groupe de Harvard, ou déduites de notre détermination de $\alpha$ par le calcul des corrections radiatives. On a porté en abscisse les trois derniers chiffres de $a_{e}$, notés xxx dans l'expression $a_{e}=0,001159652$ xxx. L'accord entre les résultats des deux groupes n'est satisfaisant (à moins de $2 \sigma$ ) que si l'on tient compte des corrections muoniques dans le calcul.

satisfaisant à condition de tenir compte non seulement des termes électroniques dans le calcul des corrections radiatives, mais aussi des corrections muoniques, dont l'ordre de grandeur est de $10^{-9}$.

On voit donc que la comparaison de ces résultats, obtenus par des méthodes expérimentales totalement différentes, permet non seulement de tester les calculs d'électrodynamique quantique, mais aussi de mettre en évidence pour la première fois la nécessité de tenir compte des corrections muoniques dans ces calculs.

\section{Conclusion}

Nous avons donné ici deux exemples de mesures menées au sein de notre équipe sur des systèmes atomiques simples. Dans les deux cas, il s'agit de mesures de fréquences optiques : mesures absolues dans le cas de la spectroscopie de l'atome d'hydrogène ; mesures relatives, c'est-à-dire décalage de fréquence dû à l'effet Doppler, dans celui de l'effet de recul de l'atome de rubidium. Nos résultats permettent de tester les prédictions de l'électrodynamique quantique sur les corrections radiatives qui affectent les propriétés soit d'un électron au sein d'un atome, soit d'un électron libre pour ce qui concerne son moment magnétique. Il est remarquable de constater que ces expériences réalisées dans un domaine d'énergie faible, de l'ordre de l'électron-volt, sont suffisamment précises pour tester, en dehors des grands équipements, des effets de la physique des hautes énergies, comme la structure du proton ou les corrections radiatives jusqu'à leurs contributions muoniques.

\section{Références}

1 - J.-C. Pain, « Willis Eugene Lamb (1913-2008), la passion de la précision », Reflets de la Physique, 36 (2013) 27-29.

2. A. Amy-Klein, « Des lasers à impulsions femtosecondes pour mesurer les fréquences ", Reflets de la Physique, 21 (2010) 42-45.

3• C.G. Parthey et al., "Improved measurement of the hydrogen 1S-2S transition frequency", Phys. Rev. Lett. 107 (2011) 203001.

4 O. Arnoult, F. Nez, L. Julien et F. Biraben, "Optical frequency measurement of the 1S-3S two-photon transition in hydrogen", Eur. Phys. J. D60 (2010) 243.

5• A. Antognini et al., "Proton structure from the measurement of $2 \mathrm{~S}-2 \mathrm{P}$ transition frequencies of muonic hydrogen", Science 339 (2013) 417.

6• H. Fleurbaey et al., "New measurement of the 1S-3S transition frequency of hydrogen: contribution to the proton charge radius puzzle", Phys. Rev. Lett., 120 (2018) 183001.

7. D. Hanneke et al., "New measurement of the electron magnetic moment and the fine structure constant", Phys. Rev. Lett. 100 (2008) 120801

8- A. Landragin et F. Pereira dos Santos, "Le laser: un outil de choix pour l'interférométrie atomique ", Reflets de la physique 21 (2010) 53-57.

9• R. Bouchendira et al., "New détermination of the fine structure constant and test of the quantum electrodynamics", Phys. Rev. Lett. 106 (2011) 080801. 